\title{
Curvature of the spine in post-encephalitic Parkinsonism
}

\author{
J. PURDON MARTIN \\ From the National Hospital for Nervous Diseases, Queen Square, and the Highlands Hospital, \\ Winchmore Hill, London
}

Some degree of lateral curvature of the spine is common in post-encephalitic Parkinsonism, and in a proportion of the cases the body becomes markedly bent over to one side or the other. Although there is almost invariably some degree of antero-posterior deformity, the appearance from behind when the patient is standing or sitting usually shows an almost uniform lateral curve of the spinous processes, extending from sacrum to neck (Fig. 2) but in some instances concentrated in the mid-dorsal region (Fig. 4). The head is usually flexed in the same direction as the trunk, but in some cases its tilt is to the other side.

The patients become unable to hold their bodies erect while sitting, and fall over to what I will call the 'affected' side. When standing and walking they may still be able to preserve their equilibrium but as time and the disease proceed' they become so liable to fall that movement without a supporting or steadying arm is too perilous.

One's first thought on observing such a patient is that the upper part of the body is drawn down by hypertonus (spasm) of the muscles, especially the long muscles, of the corresponding side, but closer examination shows, more often than not, that the muscular rigidity is greater on the other side. Of 14 patients with pronounced lateral scoliosis of the kind described here, eight are inclined to the side of lesser rigidity, four to the side of greater rigidity, and in the other two, in both of whom the curvature is pronounced, there is no significant difference in the degree of rigidity on the two sides, nor does the clinical history indicate that there was such a difference at any stage.

\section{CASE REPORTS}

CASE 1 S.S., a woman, born in 1895, had 'influenza' with double vision in 1926, and a few months later she felt that her body was stiffening up and her hands began to shake, the left being always much worse than the right; salivation was troublesome and swallowing difficult.

About 1946, following a fall, she noticed that her body was becoming bent over to the right side, and this was followed soon afterwards by a similar bending of the neck to the same side. She began to have severe pain in the right side of her body under the ribs; this was worse when she was standing, and she derived no benefit from physiotherapy, corrective exercises, or a spinal support. By 1949 she was unable to walk because she fell over to the right. In March 1950 an operation was performed for removal of the tenth and eleventh ribs on the right side and this relieved her pain. At that time her head and body were flexed to the right by a gross degree of midthoracic scoliosis (Fig. 1). Radiological examination, confirming this, showed some rotation of the vertebral bodies and secondary osteoarthritic changes.

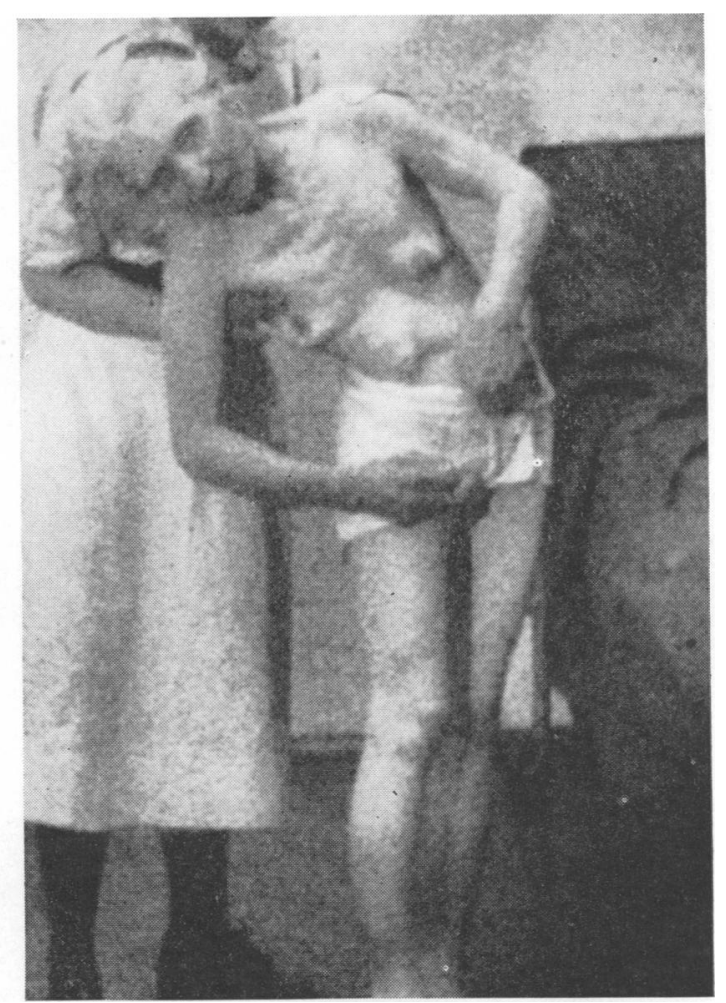

FIG. 1. Case 1. The patient in 1950. Scoliosis concave to the less rigid side. 
The pain was attributed to 'crowding' of the right lower ribs against the iliac crest. It was completely relieved by the operation; however, within a few months some pain returned, the next rib now pressing against the iliac crest. The patient died in 1957.

CASE 2 V.H., a woman, born in 1911, had encephalitis in 1925. Stiffness began in the left hand about three months after the acute illness and gradually got worse. Now the left arm is held strongly flexed and adducted, the left wrist is strongly flexed and cannot be straightened; the fingers are flexed at the basal joints and hyperextended. The left leg is partly flexed at the knee and extended at the ankle and the patient walks on the ball of the foot. There is no rigidity of the right side. The patient is right-handed and does embroidery and typing, and her right leg acts normally in walking. Her spine is curved to the right (Fig. 2); she is round-shouldered and holds her head forward and to the right. When she is blindfolded her head falls forward. She is dysarthric and has considerable dysphagia.

$X$-ray films of the spine show that in spite of the uniformity of the external appearance the curvature is most pronounced in the dorso-lumbar region.

CASE 3 This patient, G.D., is extremely deformed and completely disabled.

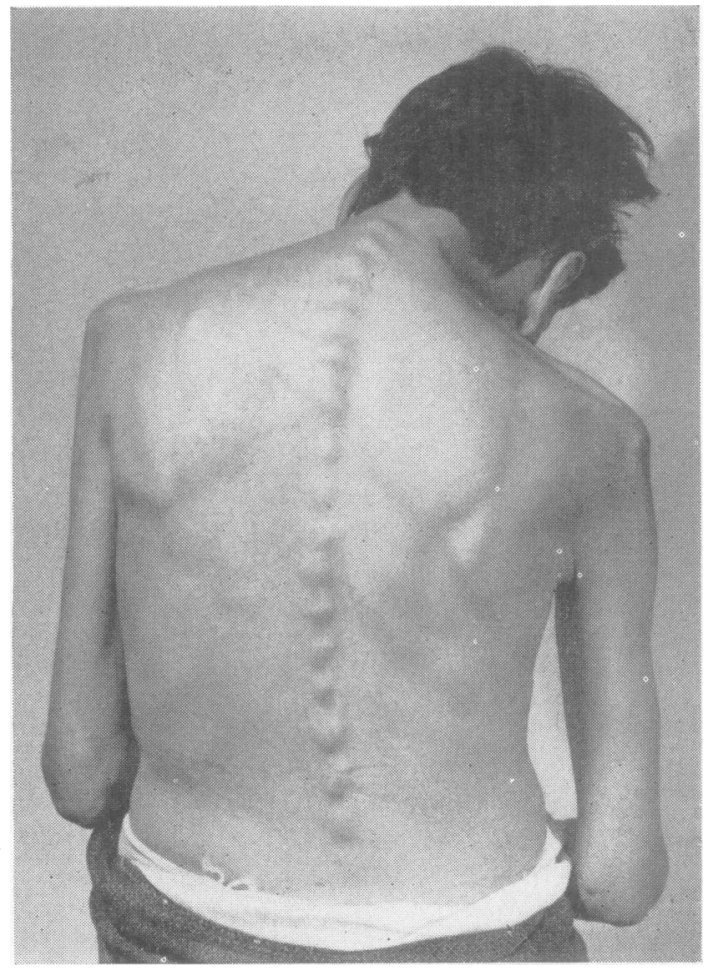

FIG. 2. Case 2. Uniform curvature concave to the right in spite of severe rigidity in the left side.

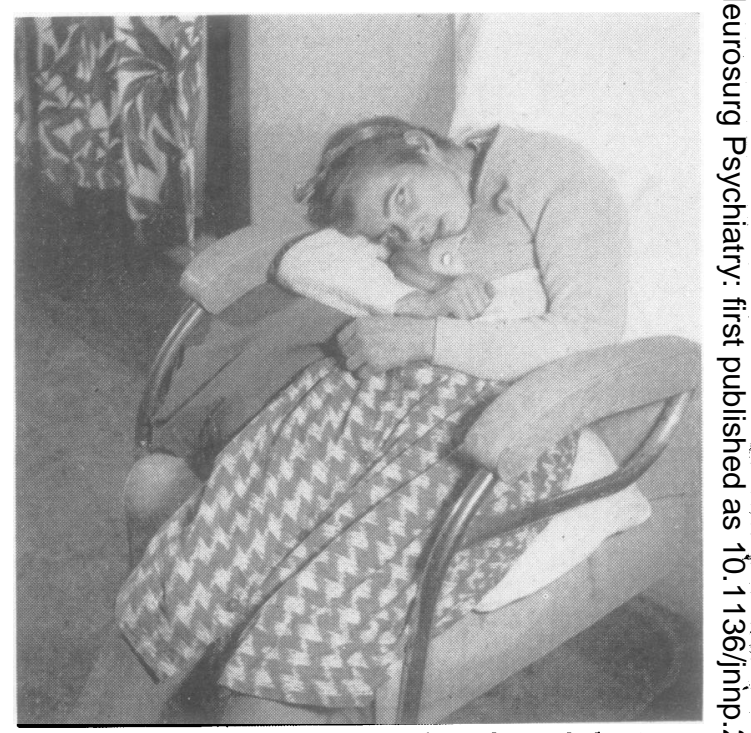

FIG. 3. Case 3. Severe flexion to the right, with dominance of rigidity on the right side.

Born in 1919, she had encephalitis as a child. Her on serviceable limb is her left arm and hand. Rigidity severe in the other three limbs, and is perhaps wors 0 in the right hand. This hand is held dorsiflexed at the wrist with the fingers flexed at the basal and proximg intrinsic joints, and hyperextended at the last joints $\frac{\mathbb{Q}}{-}$ As seen from the front her body and head appear to to bent over through more than a right angle.

CASE 4 L.H., a woman, born in 1907, had encephalitis in 1924 and began to shake soon afterwards. In 1961, she complained of 'attacks' of very bad shaking, and that

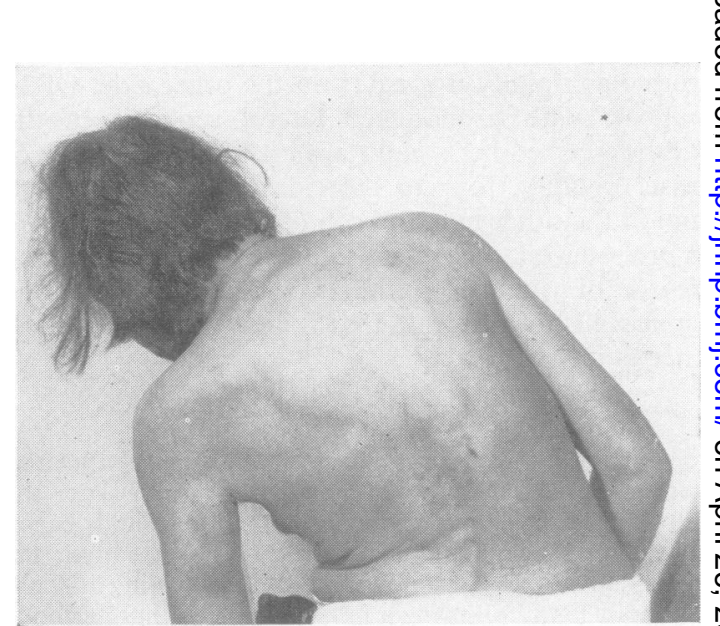

FIG. 4. Case 4. Lateral curvature concentrated in the dorso-lumbar region.

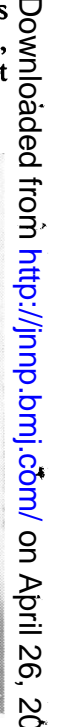
N 


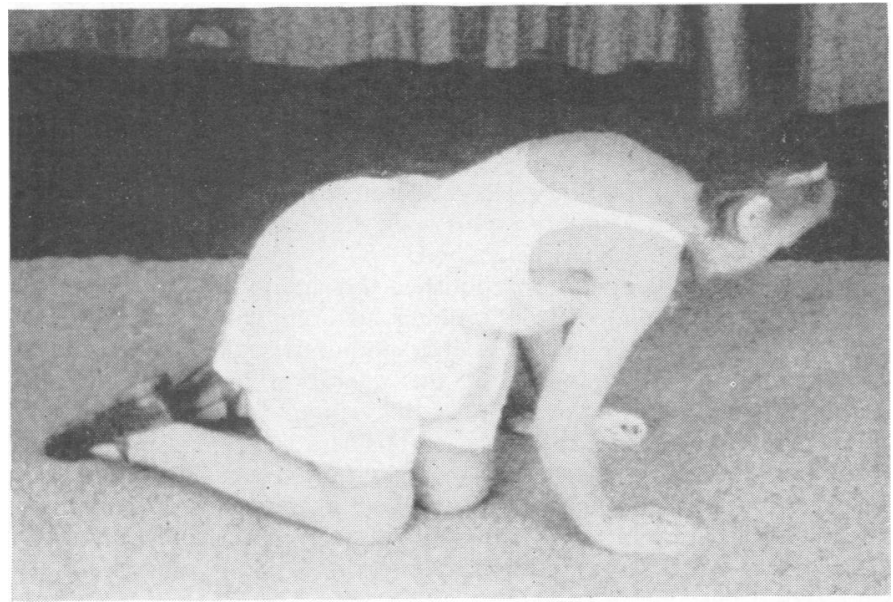

FIG. 5. Case 4. The patient crawling. The body is deviated to the left, and the fore-limbs and hind limbs appear to be going in different directions.

when sitting in a chair she fell over to the left. She has considerable rigidity of all her limbs and there is no significant difference in its intensity on the two sides. Her neck is rigid and passive rotation meets with more resistance to the right than to the left. She has tremor of both hands, approximately equal in both. She is unable to rise from an ordinary chair, but she walks well, with a strong tilt to the left, and she has a tendency to fall backwards and to the left. She has marked scoliosis with the curve of the spinous processes concave to the left, and most pronounced in the lumbo-dorsal region, and the right half of the chest is the more prominent behind; the pelvis is straight. $X$-ray films taken in the standing position show that the flexion of the spine is concentrated about the dorso-lumbar junction; in the lying position the deformity can be largely corrected.

On all fours the line of her hands is not at all parallel to the line of her knees, and when she crawls the movement of her knees is to a great extent lateral. Crawling along a carpet she deviates to the left, especially when blindfolded; she turns easily to the left and has great difficulty in turning to the right. (Fig. 5).

CASE 5 I.K., a man, born in 1905, has a vague history of illness when aged between 16 and 18 .

The diagnosis of Parkinson's disease was made in 1952; it had begun in the left side. By 1956 he had severe muscular rigidity in the left arm and moderate rigidity in the left leg. The right side was normal. When seen in 1957 he walked with his body inclined to the right and he had raised the heel of his right shoe and lowered that of the left. The left arm was very rigid and rigidity had increased in the left leg. He had no definite rigidity in the right arm, but his writing had become small. His speech was monotonous. In 1958 he began to use a high walking stick (Moses staff) in his right hand to support his right side, and he complained of pain in his back, which he attributed to the flexion of his spine. When he was radiographed in the supine position the vertebral column was straight and the films showed no abnormality. A high surgical jacket was ordered but he found it very uncomfortable and soon discarded it. By 1962 he was still more bent over to the right and when sitting he tended to fall to the right. He was now unable to rise from a chair and when standing was easily displaced backwards. He could not rise from the floor. He could turn over when lying on the floor, but said he could not turn over in bed and could not get out of bed without assistance. When tilted slightly in the sitting position he fell over at once to the right; he showed no reaction of his body nor of the limbs of the right side against the tilt, but some reaction in the limbs of the left side. In September 1962, he underwent a stereotactic operation (Mr. Gillingham), and lesions were placed in the right ventro-lateral thalamus and in the right pallidum. His convalescence was slow, and when he began to get up he was very unstable and fell repeatedly to the left. A year later he stood almost straight, though his right shoulder was still lower than the left. He said he felt the operation had been worth while because it has straightened him up and relieved the pain in his back. In his left arm there was very slight rigidity and no tremor, but he still carried this arm in the 'hemiplegic' posture; he said he could not do much with his left hand, but he was training himself. He was no better at dressing or in using a knife or fork. His speech was still very mumbling. His postural reactions were unchanged, and he fell several times daily, always to the left.

Two years after the operation he showed a mere suspicion of flexion to the right. He had no rigidity of the left arm or tremor of the left hand and said that his left side was good. Alternating movements were still very poor. He had tremor of the right hand and moderate rigidity in the right arm, and he said that his left hand was now stronger than his right. He walked well, though not swinging his arms, and he still had some tendency to fall to the left. When standing he was easily displaced backwards. Now, he could rise from a chair quite easily. However, he could not rise from the floor, and he had difficulty in turning over to his left; he could not rise to the 'all-fours' position, but when placed in that position he could crawl well. On the tilting apparatus he showed, 
as before, some reaction in the left limbs, but none in the right; he fell over more easily to the left than to the right.

By now the effects of the operation were as follows:It had abolished the flexion of his spine. It had abolished the rigidity and tremor in his left side. He walked better. He had regained the ability to rise from a chair. He was liable to fall to the left. His postural disabilities were otherwise unchanged.

CASE 6 J.B. was born in 1910. He had encephalitis when a schoolboy.and was admitted to hospital soon afterwards because of 'brain storms'. His left side gradually became very rigid; the arm was held strongly adducted, flexed at the elbow and wrist with the fingers extended; the hand was subject to gross tremor. His body became bent over to the right, and as he walked it bent forward rhythmically when it was supported on his left leg.

In 1956, a right-sided stereotactic operation directed towards the pallidum was performed by Mr. McCaul. The rigidity and tremor of the left arm and hand were abolished and the flexion spasms during walking were greatly reduced and soon died out. His body gradually became much straighter and it has remained so, though its posture has not become quite normal. Some tremor has returned to the left hand but there is no significant rigidity.

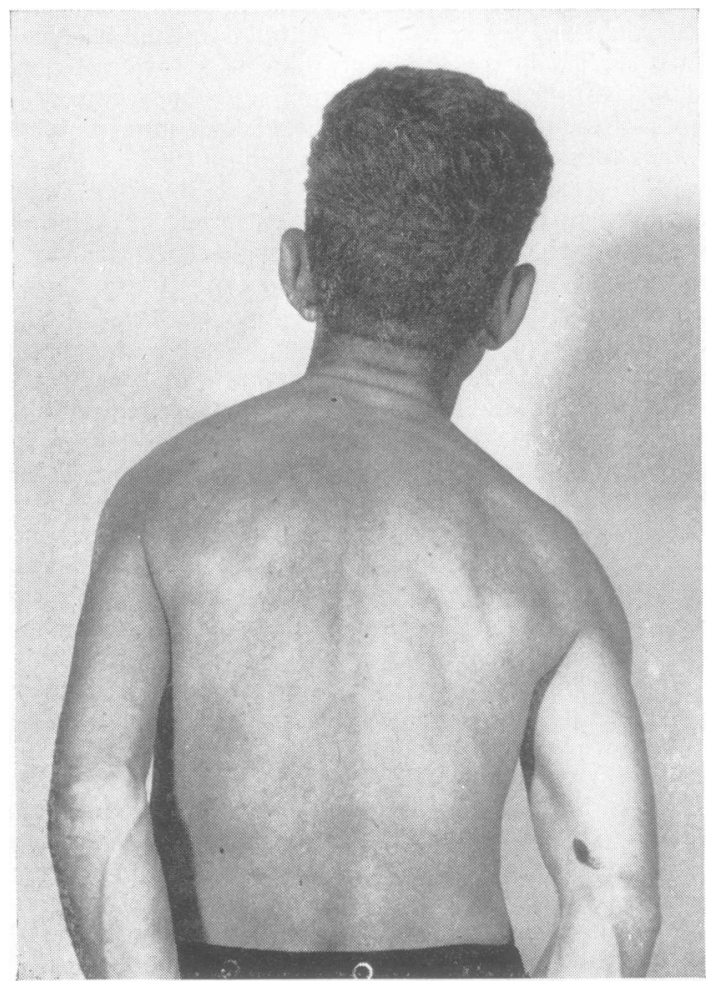

FIG. 6. Case 6. The patient eight years after a stereotactic operation on the right side of the brain; his body was bent over to the right, in spite of severe rigidity on the left.
CASE 7 E.H., a man, born in 1908, had an illness in 1926, and afterwards his eyes used to turn up. Some years later his left side became stiff, and eventually his left hand was more or less fixed. In 1957 he had a stereotactic operation (Mr. McCaul) directed towards the right pallidum and at that time his left arm and leg were very rigid. His body was moderately bent over to the left. He had no rigidity of his right side and he swung his right arm a little as he walked.

The operation abolished the rigidity and tremor in his left side. Seven years later his body is moderately flexed to the left; he says it is about the same as before the operation, and that the operation had no noticeable effect on the state of his spine. He has no rigidity in either arm, but has considerable tremor in his right hand and some in his right foot. He walks well and is generally active. On the tilting apparatus he shows no movements of the body or lower limbs and only some variable responses in the arms.

These cases suffice to demonstrate the variations of the symptom and the absence of any consistent clinical relation to rigidity or tremor.

Case 4 displays an additional clinical feature in the patient's liability to deviate to the concave side wheno crawling, an effect which was more pronounced, when she was deprived of visual guidance. Associa ted with this was an obvious difficulty in turning against the curvature. Probably both of these effects are to be explained by purely physical circumstances and the patient's inability to control them. In ordef to turn to the right the subject's centre of gravity must be moved to the right of the middle line, since the $\overrightarrow{0}$ body must tend to fall towards the desired side. The of deflection of the body to the left brings the centre of gravity to the left of the middle line and makes it easy for the subject to turn to the left, and any difficulty in making the postural adjustments necessary to move it over to the right makes it correspondingly difficult for her to turn to the right. Her tendency to fall to the left when standing shows that control of her centre of gravity is impaired.

\section{DISCUSSION}

There can be little doubt that this lateral spinal curvature is due to the disease, and so, to a central nervous disturbance, and probably one involving the basal ganglia. This view is confirmed by the fact that it can be influenced by a stereotactic operation which places lesions in the globus pallidus or its fibre connexions.

The curvature, especially when it is away from the side of greater muscular tone, cannot be simply a passive process, a negative symptom resulting from some loss of muscular function. Also, it is not due to spinal disease, as is shown by the radiological 
appearances and by the reversibility of the deformity. The only satisfactory explanation is that it is due to a positive neuromuscular activity affecting the posture.

Again, since the curvature may be either against or with the balance of rigidity the observations provide no evidence that it is either directly due to the rigidity or on the other hand that it is a reaction to the rigidity, or is in any way dependent on it. Further, more, in many other cases of Parkinsonism, both idiopathic and post-encephalitic, there is a considerable difference in the rigidity of the two sides and yet no significant scoliosis develops. Apart from their occurrence in the same disease, the two symptoms appear to be unconnected.

Cases 5 and 6 demonstrate the effects of operations which were ipsilateral to the concavity, and case 7 those of a contralateral operation. In all these cases the operations abolished the rigidity and tremor on the opposite side of the body, showing that the surgical lesions were satisfactorily placed. When the operation was ipsilateral to the concavity (cases 5 and 6) it apparently relieved the cause of the curvature as well, but when it was contralateral to the concavity (case 7) it did not do so. The positive effect is the more important, and it indicates that the central nervous activity which is responsible for the curvature is on the same side as the concavity of the spinal curve, and the evidence of the negative result, as far as it goes, is in the same sense.

Under various experimental conditions curvature of the spine, and so of the trunk, to one side or the other, have been described in different species of animals, but usually in quadrupeds. The work that is most relevant to the present enquiry is that of Delmas-Marsalet (1925) who, working with dogs, found that severe lesions of the head of the caudate nucleus gave rise to a disturbance of posture in which the body and head were strongly flexed laterally, with the concavity to the side of the lesion. The animals circled to the side of the concavity and were unable to turn to the other side. Electrical stimulation of the same nucleus gave rise to a contralateral effect, i.e., the concavity was to the opposite side, and Delmas-Marsalet mentioned that Ferrier (1876) had obtained a similar result. More recently Hassler (1956), reviewing Hess's material, has described the same effects in freely moving cats. Delmas-Marsalet further found that when destruction of the head of the caudate on one side had caused deflection of the body to that side, destruction of the head of the other caudate nucleus caused the body to straighten again. This suggests that under normal conditions (in the dog) there is a balance of activity between the two caudate nuclei.

Delmas-Marsalet considered that the lesion of the caudate released a postural activity ('Magnus and

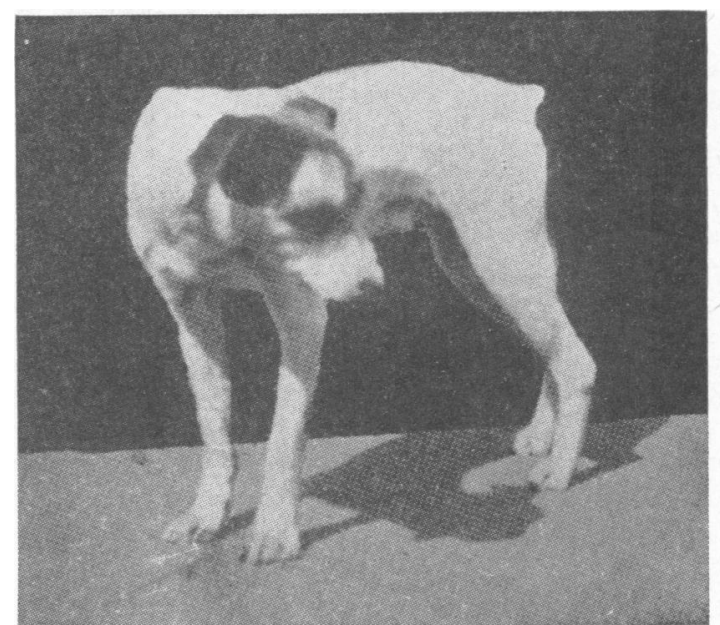

FIG. 7. Dog after unilateral caudate lesion, showing curvature of the spine and turning of the head to the side of the lesion (after Delmas-Marsalet).

de Kleyn reflex') which produced the curvature of the body.

In many cases of post-encephalitic Parkinsonism, the head of the caudate nucleus is severely involved in the pathological process (Martin, 1965) and in cases where such lesions are unilateral, or predominantly so, it is likely, in view of these experimental results, that an effect similar to that described by Delmas-Marsalet in the dog would occur. According to present views, the influence of the caudate on the pallidum is a controlling one, and so the curvature of the body would be more immediately attributable to an excessive activity of the pallidum consequent upon the damage to the head of the caudate. It is this excessive or unbalanced activity which would be amenable to abolition by an operation which placed a lesion either in the pallidum itself (as in the earlier operations) or in its efferent pathways.

The same operation by its effect on another excessive activity of the pallidum abolishes or relieves the rigidity in the contralateral half of the body. There is considerable evidence (Denny-Brown, 1962) which permits us to attribute such rigidity to lesions of the putamen, and putting the two together, it seems that curvature of the spine to one side, with the greater rigidity on the other, is likely to occur when disease has affected predominantly the caudate and putamen of one side.

\section{SUMMARY}

Cases of post-encephalitic Parkinsonism are described in which the body becomes severely bent over to one side. 
The body may straighten up again after a sterotactic operation on the side of the brain ipsilateral to the concavity of the scoliosis.

It is suggested that the condition is homologous to that produced experimentally in dogs by destruction of one caudate nucleus, and that the clinical state is due to a severe imbalance of the activity of the two caudate nuclei.

\section{ADDENDUM}

Of five similar post-encephalitic patients observed by Onuaguluchi (1964) three were bent towards the less rigid side and one to the more rigid, and in the case of the fifth patient the lateral preponderance was not definite.

I am indebted to Dr. Joseph Sharkey, the Medical Superintendent, and to the nursing staff of the Highlands
Hospital, for their cooperation in respect of cases 2, 3, 4, 6, and 7, and to Mr. Prickett and his staff in the Department of Medical Illustration of the Institute of Neurology, for preparing the illustrations. Figure 1 is from a cinematograph film kindly lent by Dr. J. A. V. Bates.

My thanks are due also to the Parkinson's Disease Foundation of America for a grant to meet the expenses of this and related work.

\section{REFERENCES}

Delmas-Marsalet, P. (1925). Contribution experimentale à l'etude des Functions du Noyau caudé. Thèse, Bordeaux.

Denny-Brown, D. (1962). The Basal Ganglia. Oxford University Press, London.

Ferrier, D. (1876). The Functions of the Brain. Smith, Elder and Co., London.

Hassler, R. (1956). Die zentralen Apparate der Wendebewegungen, II Arch. Psychiat. Nervenkr., 194, 481-516.

Martin, J. Purdon (1965). The Globus pallidus in post-encephalitic Parkinsonism. J. neurol. Sci., 2, 344-365.

Onuaguluchi, G. (1964). Parkinsonism. Butterworths, London. 